\title{
Role of Vibrations in Orthodontics: A Review
}

\author{
Dr Varun Goyal,' Dr Raj Kumar Singh,, Dr Nishant Gupta, ${ }^{3}$ Dr Gurkeerat Singh,, ${ }^{4}$ Dr Ashima Relhan \\ ${ }^{2.3}$ Reader, ${ }^{4}$ Professor and Head of the Department, ${ }^{5}$ Assistant Professor, \\ Sudha Rustagi College of Dental Sciences and Research \\ Faridabad, Haryana, India
}

Correspondence: Dr Raj Kumar Singh; Email: rajortho2010@gmail.com

\section{ABSTRACT}

Long treatment time has been a deterrent for the patients for pursuing orthodontic treatment. Orthodontist from time immemorial have been trying different methods for reducing the treatment time. It has been documented in literature that vibration therapy helps in bone remodelling and muscle regeneration as demonstrated in cases of osteoporosis, muscle loss and joint pain. Using the similar approach orthodontist from different era have tried to accelerate the tooth movement using different methods. This article summarises the efforts of different clinicians and their approaches to achieve reduced orthodontic treatment time as well as reducing the discomfort, pain and root resorption using pulsations and vibrations

\section{INTRODUCTION}

The scientists across the world have documented positive results using the vibrational therapy in patients with osteoporosis, muscle loss and joint pain. Eight years ago, NASA scientists reported that muscles atrophies relatively quickly and bones lose mass on prolonged exposures to weightlessness. Therefore, they suggested their astronauts to stand on a lightly vibrating plate for 10 to 20 minutes each day while in orbit so as to prevent bone loss.

\section{VIBRATIONS \& TOOTH MOVEMENT}

Traditional orthodontic treatment requires an average of 24 months, so the possibility of shortening this will be welcomed by both patients and clinicians. Pharmacological approaches have been employed (injection of prostaglandins and peptide hormones around the teeth) but they have all come along with different sets of problems, such as pain, severe root resorption and drug-induced side effects. ${ }^{1,2}$ Vibration therapy has been used in health care since the 1800s. Advanced molecular biology techniques have shown the researchers new avenue towards finding answers to the questions asked for the last few decades. ${ }^{3}$ The rate of tooth movement has been enhanced by pharmaco-therapeutic or electrophysiological means. The applied electrical fields can alter the normal electrical states of bone and cartilage, induce increased rates of cellular division and metabolism, and thus promote increased healing of bony and cartilaginous defects. ${ }^{4,5}$ The application of an electric current may alter the electrolytic environment allowing changes in the type and rate of ions $(\mathrm{K}+, \mathrm{Ca}++$ etc) that move across the cell membranes \& acts as a mediator for cellular changes. Micro-pulsed electrical stimulation could reach bone osteoblasts non-invasively and can result in an increase in the CAMP and CGMP. These cyclic nucleotides are a type of second messenger, which play a role in the efficient remodelling of alveolar bone and tooth movement. ${ }^{3}$

The first known attempt to apply pulsating forces to the dentition in an orthodontic application with humans was likely conducted by Everett Shapiro et al. ${ }^{2}$ Davidovitch et al suggested orthodontic tooth movement may be accelerated by the use of locally applied electric currents. ${ }^{6}$ The rate of movement as well as the total movement was found to be greater with the pulsed tooth than in the control tooth with the rate of movement being two-fold higher at times. $2,7,8$ In a monkey model, tooth movement rates up to $40 \%$ faster were demonstrated in response to vibration as early as 1986 by Shimizu et al.9.10 More recently, $\mathrm{H}$. Utomo (Airlangga University) has suggested via literature search that patients who regularly chews gum (a crude form of vibratory force application) exhibit accelerated rates of tooth movement. It is believed that orthodontic tooth movement is accompanied by "site-specific" alveolar bone remodelling \& is essential for tooth movement." It is characterized by tandem periods of osteoclastic recruitment, bone resorption, reversal and bone formation. ${ }^{12,13}$ This process involves 
the periodontal ligament and is dependent on the magnitude and consistency of the force being applied. In the area of periodontal ligament compression, osteoclasts proliferate and initial resorption of superficial bone occurs. ${ }^{13-15}$ In the region of periodontal ligament tension, the periodontal fibres unwind, fibroblasts appear and osteoblasts form a non-mineralized collagenous matrix called osteoid. The osteoid is later mineralized, trapping some osteocytes in lacunae within the bone. ${ }^{16}$ A study by Davidovitch et al results suggests that electric stimulation enhances cellular enzymatic phosphorylation activities in periodontal tissues and may be a potent tool in accelerating alveolar bone turnover. ${ }^{17}$ According to Jafar Kolahi, direct electric current is a potent biologic mean to accelerate periodontal tissue turnover and orthodontic tooth movement. ${ }^{18}$ In 1978 Meikle et al developed organ culture systems in which mechanical force could be applied to rabbit cranial sutures under controlled conditions. ${ }^{19}$ This simple experimental model mimics the forces to which the periodontal ligament and other sutural articulations of the craniofacial skeleton are exposed during orthodontic treatment. Kopher and Mao assessed peak cyclic forces of $5 \mathrm{~N}$ magnitude at $1 \mathrm{~Hz}$ in rabbits while Peptan and Mao assessed cyclic forces of $1 \mathrm{~N}$ at $8 \mathrm{~Hz}$ in rabbits and $\mathrm{Vij}$ and Mao assessed cyclic forces of $300 \mathrm{mN}$ at $4 \mathrm{~Hz}$ in rats. In aggregate, the data from these three studies indicate that cyclic forces between $1 \mathrm{~Hz}$ and $8 \mathrm{~Hz}$ with forces ranging from $0.3 \mathrm{~N}$ to $5 \mathrm{~N}$, increase bone remodelling..$^{20,21}$ Jeremy Mao is the inventor of the concept behind vibrating force to enhance and accelerate tooth movement. A number of different devices have been marketed which use various means of vibrating the teeth.

In early 2008, a confirmatory paper was published in the American Journal of Orthodontics and Dentofacial Orthopaedics out of Tohoku University, Sendai, and Japan using a rodent model. The results were clear. The treatment group received cyclic forces once per week for just eight minutes and showed significantly accelerated tooth movement when compared to the control group. ${ }^{10}$ A physical force has five fundamental properties: magnitude, direction, point of application, duration, and frequency. As orthodontists, we have studied all of these characteristics extensively with the exception of frequency. A study by Darendeliler (2001), suggested that the PEMF-induced vibration may enhance the effect of mechanical and magnetic forces on tooth movement. ${ }^{22}$

\section{BONE BIOLOGY}

Some reports described asymmetric voltage waveforms from mechanically deformed live bone. These changes were presumed to occur in bone during physical activity as a result of mechanical forces. The polarity depends on the direction of bending. Areas under pressure will be in an electropositive state \& are usually associated with osteoclastic activity and areas under tension will be in an electronegative state \& are associated with osteoblastic activity. Hence the associated area of alveolar bone is associated with both increased osteoblastic activity and osteoclastic activity. The application of cyclic loading (controlled vibrations) will not only increase the rate of tooth movement but will also create a solid foundation of bone and adjacent tissues in the mouth. ${ }^{23,24}$

\section{HISTORY OF VIBRATIONAL FORCE TO ACCELERATE BONE REMODELLING}

Based on Wolff's Law of bone remodelling and literature from long bone biology it becomes evident that vibration could accelerate bone density formation. Bone remodelling can be significantly accelerated via the application of pulsating force of various frequency and magnitude combinations. ${ }^{7.8}$ It is believed that cyclic forces increase the cellular signalling that regulates bone remodelling thus enhancing the rate of orthodontic tooth movement. Factors that increase the rate of bone remodelling have been shown to increase the rate of tooth movement. Bassett proposed that tissue integrity and function could be restored by applying electrical and/or mechanical energy to the area of injury. ${ }^{25}$ Previous animal and clinical studies have shown that electrical \& mechanical stimulation, in particular low intensity pulsed ultrasound, when applied to the non- healing fractures, improved the rate of bone healing via up-regulation of cartilage formation and maturation of endochondral bone formation. ${ }^{16}$

In a number of studies it has been shown that prostaglandins are involved in the bone removal component of orthodontic tooth movement. In addition, inhibitors to prostaglandin production (cyclooxygenase or COX inhibitors) are known to decrease the amount of orthodontic tooth movement. Parathyroid hormone (PTH) is a potent bone-remodelling factor. Continuous infusion of PTH has been shown to cause a two fold increase in the rate of orthodontic tooth movement in rats. $^{1,2}$ 


\section{VIBRATION TO CONTROL PAIN}

A device that vibrates at frequency (between $100 \mathrm{~Hz}$ and $250 \mathrm{~Hz}$ ) and at a force (approximately $100 \mathrm{~g}$ or 1N) has been commercially distributed and used in humans for reducing pain associated with orthodontic adjustments. Marie, et al reported on 48 patients who experienced significant reduction in pain; no adverse events were reported. ${ }^{26}$

\section{VIBRATION AND ROOT RESORPTION}

Nishimura et al studied $60 \mathrm{~Hz}$ vibrations in a direct tooth movement model in rats. Rats received "standard orthodontics" through application of a spring that applied a force between the molars. Vibration to the molars at a $60 \mathrm{~Hz}$ frequency was added through a separate apparatus. The group that received vibration had a statistically significant increase in tooth movement when compared to the spring-force (static force) only group. Interestingly, the vibration group had a trend toward less root-resorption as compared to the static force-only group. ${ }^{27}$

\section{VIBRATIONAL EFFECT ON BONE ARCHITECTURE}

Research has demonstrated that the use of cyclic forces increases the rate of bone remodelling compared to static forces. ${ }^{13,28,29}$ In a pilot study in one human subject, a pulsating force device was investigated and was found to enhance and speed tooth movement, although it was never introduced commercially; both the rate of movement and the total amount of movement were enhanced. Cyclic forces have been found to accelerate the rate of

bone remodelling to levels far greater than static forces or intermittent forces. ${ }^{30-33}$ While similar in their nonconstant nature, cyclic forces- sometimes referred to as pulsatile forces, are different than intermittent forces that are applied for some duration of time, removed, and then reapplied. ${ }^{34}$ A static force occurs once and affects cells once; an intermittent force is still a static force, the only difference is that it is introduced episodically. In contrast, cyclic forces are oscillatory in nature and change magnitude rapidly and repeatedly, affecting the cells with each oscillation of force magnitude. ${ }^{34,35}$ The frequency of cyclic forces is never zero. Force frequency is a concept of critical importance, but has rarely been considered in the field of orthodontics and Dentofacial Orthopedics until recent years. Cyclic forces cause deformation by changing a structure's length multiple times, whereas intermittent and static forces can only do so once per application. At force frequencies that are greater than zero, cells are impacted multiple times. Frequencies of interest for orthodontic application range from several hertz (Hz.) up to $100 \mathrm{~Hz}$. or more. Cyclic forces impact tissue structures and cells multiple times, and this seemingly subtle difference has been shown to lead to dramatic differences in biological response in both orofacial and long bones.35,36 Multiple cycles of change in force magnitude or cyclic forces are significant because cells respond more readily to rapid oscillation in force magnitude than to constant force. ${ }^{36}$

A force propagating through a biological tissue, such as alveolar bone and the periodontal ligament, is transduced as a tissue-borne and cell-borne mechanical stress that in turn induces interstitial flow. ${ }^{37}$ Although fluid flow is a current focus of the mechanotransduction pathways, its anabolic and catabolic effects rely upon deformation of extracellular matrix molecules, transmembrane channels, the cytoskeleton and intranuclear structures. ${ }^{36-38}$ Cells are known to respond more readily to rapid oscillation in force magnitude (i.e. to cyclic forces) than to constant forces. ${ }^{37}$ Animal studies using cyclic forces of 0.3-5 newtons ( $N$ ) have demonstrated increased bone remodelling and the delivery of cyclic forces by a vibrational device applied to molar teeth in the presence of standard static forces from an orthodontic spring resulted in a significant increase in tooth movement compared to no adjunctive device use. There was also a trend towards less root resorption when cyclic forces were applied. ${ }^{20,21,27}$ Cyclic forces have been used for other parts of the body, such as the Juvent system that is used to counteract lost bone and muscle. A second device using cyclic forces was introduced to relieve the discomfort associated with orthodontic adjustments and was found to be safe and effective. ${ }^{26}$

\section{CONCLUSION}

Short durations of extremely small magnitude, highfrequency mechanical stimuli can promote anabolic activity in the adult skeleton. ${ }^{39}$ Certain exercises can induce osteogenesis and improve bone strength. Experimental protocols that insert "rest" periods to reduce the effects of desensitization can double anabolic responses to mechanical loading. ${ }^{39-41}$ Recovery periods restore mechanosensitivity to dynamically loaded bone Here, it is determined that 
whether such signals can influence trabecular and cortical formative and resorptive activity in the growing skeleton; is the newly formed bone of high quality and would the insertion of rest periods during the loading phase enhance the efficacy of the mechanical regimen. Site specifically attenuate the declining levels of bone formation and maintain a high level of matrix quality. If WBV prove to be efficacious in the growing human skeleton, they may be able to provide the basis for a non-pharmacological and safe means to increase peak bone mass and ultimately reduce the incidence of osteoporosis or stress fractures later in life. ${ }^{42}$

\section{REFERENCES}

1. Chao CF, Shih C, Wang TM, Lo TH. Effects of prostaglandin E2 on alveolar bone resorption during orthodontic tooth movement. Cells Tissues Organs. 1988;132(4):304-9.

2. Shapiro E, Roeber F, Klempner L. Pulsating forces in orthodontics. American Journal of Orthodontics. 1981 Mar 1;79(3):348.

3. Norton LA, Rodan GA, Bourret LA. Epiphyseal cartilage cAMP changes produced by electrical and mechanical perturbations. Clinical orthopaedics and related research. 1977 May 1;124:59-68.

4. Martin RB, Gutman W. The effect of electric fields on osteoporosis of disuse. Calcified Tissue International. 1978 Dec 1;25(1):23-7.

5. Bassett CA. Beneficial effects of electromagnetic fields. Journal of cellular biochemistry. 1993 Apr 1;51 (4):387-93.

6. Davidovitch Z, Finkelson MD, Steigman S, Shanfeld JL, Montgomery PC, Korostoff E. Electric currents, bone remodeling, and orthodontic tooth movement: II. Increase in rate of tooth movement and periodontal cyclic nucleotide levels by combined force and electric current. American journal of orthodontics. 1980 Jan 1;77(1):33-47.

7. Sato Y. Effects of bisphosphonate administration on root and bone resorption during experimental movement of rat molars. Biological Mechanisms of Tooth Movement and Craniofacial Adaptation. 2000:243-52.

8. Sato Y, Sakai H, Kobayashi Y, Shibasaki Y, Sasaki T. Bisphosphonate administration alters subcellular localization of vacuolar $\square$ type H+ ATPase and cathepsin K in osteoclasts during experimental movement of rat molars. The Anatomical Record. 2000 Sep 1;260(1):72-80.

9. Turner $\mathrm{CH}$, Robling AG. Mechanisms by which exercise improves bone strength. Journal of bone and mineral metabolism. 2005 Jan $1 ; 23: 16-22$.

10. Vij K, Mao JJ. Geometry and cell density of rat craniofacial sutures during early postnatal development and upon in vivo cyclic loading. Bone. 2006 May 31;38(5):722-30.

11. Kopher RA, Mao JJ. Suture growth modulated by the oscillatory component of micromechanical strain. Journal of Bone and Mineral Research. 2003 Mar 1;18(3):521-8.

12. Rubin CT, Donahue HJ, Rubin JE, MCLeod KJ. Optimization of electric field parameters for the control of bone remodeling: exploitation of an indigenous mechanism for the prevention of osteopenia. Journal of Bone and Mineral Research. 1993 Dec 1;8(S2).

13. Darendeliler MA, Zea A, Shen G, Zoellner H. Effects of pulsed electromagnetic field vibration on tooth movement induced by magnetic and mechanical forces: a preliminary study. Australian dental journal. 2007 Dec 1;52(4):282-7.

14. Midgett RJ, Shaye R, Fruge JF. The effect of altered bone metabolism on orthodontic tooth movement. American journal of orthodontics. 1981 Sep 1;80(3):256-62

15. Karanth HS, Shetty KS. Orthodontic tooth movement and bioelectricity. Indian journal of dental research: official publication of Indian Society for Dental Research. 2000 Dec;12(4):212-21.

16. Nishimura M, Chiba M, Ohashi T, Sato M, Shimizu Y, Igarashi K, Mitani H. Periodontal tissue activation by vibration: intermittent stimulation by resonance vibration accelerates experimental tooth movement in rats. American Journal of Orthodontics and Dentofacial Orthopedics. 2008 Apr 30;133(4):572-83

17. Meikle MC, Reynolds JJ, Sellers A, Dingle JT. Rabbit cranial sutures in vitro: a new experimental model for studying the response of fibrous joints to mechanical stress. Calcified Tissue International. 1979 Dec 1;28(1):137-44.

18. Yonemori K, Matsunaga S, Ishidou Y, Maeda S, Yoshida H. Early effects of electrical stimulation on osteogenesis. Bone. 1996 Aug $1 ; 19(2): 173-80$

19. Davidovitch Z. Biological mechanisms of tooth movement and craniofacial adaptation: proceedings of the international conference held at the Great Southern Hotel, Columbus, Ohio, May 8-11, 1991. Ohio State University College of Dentistry; 1992.

20. Miyajima K, Kasai R, Kahn AJ, Hayakawa T, lizuka T. Biological mechanisms of tooth movement: in vitro analysis and clinical application. The biological mechanisms of tooth movement and craniofacial adaptation. Birmingham: EBSCO. 1992:311-7.

21. Vandevska-Radunovic $V$. Neural modulation of inflammatory reactions in dental tissues incident to orthodontic tooth movement. A review of the literature. The European Journal of Orthodontics. 1999 Jun 1;21 (3):231-47.

22. Duncan RL, Turner CH. Mechanotransduction and the functional response of bone to mechanical strain. Calcif Tissue Int. 2005; 57:344-358

23. Mcleod KJ, Rubin CT, Otter MW, Qin YX. Skeletal cell stresses and bone adaptation. The American journal of the medical sciences. 1998 Sep 1;316(3):176-83.

24. Yamasaki K. The role of cyclic AMP, calcium, and prostaglandins in the induction of osteoclastic bone resorption associated with experimental tooth movement. Journal of Dental Research. 1983 Aug;62(8):877-81 
25. Rubin C, Turner AS, Bain S, Mallinckrodt C, McLeod K. Anabolism: Low mechanical signals strengthen long bones. Nature. 2001 Aug 9;412(6847):603.

26. Shimizu K, Murata H. Normal vibrations and calculated thermodynamic properties of tetramethylsilane. Journal of Molecular Spectroscopy. 1961 Jan 1:5(1-6):44-51

27. Turner $\mathrm{CH}$, Robling AG. Exercise as an anabolic stimulus for bone. Current pharmaceutical design. 2004 Aug 1;10(21):2629-41

28. Kolahi J, Abrishami M, Davidovitch Z. Microfabricated biocatalytic fuel cells: a new approach to accelerating the orthodontic tooth movement. Medical hypotheses. 2009 Sep 30;73(3):340-1.

29. Nishimura M, Chiba M, Ohashi T, Sato M, Shimizu Y, Igarashi K, Mitani H. Periodontal tissue activation by vibration: intermittent stimulation by resonance vibration accelerates experimental tooth movement in rats. American Journal of Orthodontics and Dentofacial Orthopedics. 2008 Apr 30;133(4):572-83.

30. Marie SS, Powers M, Sheridan JJ. Vibratory stimulation as a method of reducing pain after orthodontic appliance adjustment. Journal of Clinical Orthodontics. 2003 Apr;37(4):205-8.

31. Mao JJ, Wang X, Kopher RA. Biomechanics of craniofacial sutures: orthopedic implications. The Angle Orthodontist. 2003 Apr;73(2):128-35.

32. Mao JJ, Wang X, Mooney MP, Kopher RA, Nudera JA. Strain induced osteogenesis of the craniofacial suture upon controlled delivery of low-frequency cyclic forces. Front Biosci. 2003 Jan 1;8(1):a10-17.

33. Mao JJ. Calvarial development: cells and mechanics. Current Opinion in Orthopaedics. 2005 Oct 1;16(5):331-7.

34. Mao JJ. Mechanobiology of craniofacial sutures. Journal of dental research. 2002 Dec;81 (12):810-6.

35. Wang X, Mao JJ. Accelerated chondrogenesis of the rabbit cranial base growth plate by oscillatory mechanical stimuli. Journal of Bone and Mineral Research. 2002 Oct 1;17(10):1843-50.

36. Peptan Al, Lopez A, Kopher RA, Mao JJ. Responses of intramembranous bone and sutures upon in vivo cyclic tensile and compressive loading. Bone. 2008 Feb 29;42(2):432-8.

37. Mao JJ, Nah HD. Growth and development: hereditary and mechanical modulations. American journal of orthodontics and dentofacial orthopedics. 2004 Jun 30;125(6):676-89.

38. Wise GE, King GJ. Mechanisms of tooth eruption and orthodontic tooth movement. Journal of dental research. 2008 May;87(5):414-34.

39. Konoo T, Kim YJ, Gu GM, King GJ. Intermittent force in orthodontic tooth movement. Journal of dental research. 2001 Feb;80(2):457-60.

40. Gross TS, Edwards JL, Mcleod KJ, Rubin CT. Strain gradients correlate with sites of periosteal bone formation. Journal of Bone and Mineral Research. 1997 Jun 1;12(6):982-8

41. Robling AG, Burr DB, Turner CH. Recovery periods restore mechanosensitivity to dynamically loaded bone. Journal of Experimental Biology. 2001 Oct 1;204(19):3389-99.

42. Xie L, Jacobson JM, Choi ES, Busa B, Donahue LR, Miller LM, Rubin CT, Judex S. Low-level mechanical vibrations can influence bone resorption and bone formation in the growing skeleton. Bone. 2006 Nov 30;39(5):1059-66. 